\title{
A Note about the General Meromorphic Solutions of the Fisher Equation
}

\author{
Jian-ming Qi, ${ }^{1}$ Qiu-hui Chen, ${ }^{2}$ Wei-ling Xiong, ${ }^{3}$ and Wen-jun Yuan ${ }^{4,5}$ \\ ${ }^{1}$ Department of Mathematics and Physics, Shanghai Dianji University, Shanghai 201306, China \\ ${ }^{2}$ Cisco School of Informatics, Guangdong University of Foreign Studies, Guangzhou 510420, China \\ ${ }^{3}$ Department of Information and Computing Science, Guangxi University of Technology, Liuzhou 545006, China \\ ${ }^{4}$ School of Mathematics and Information Science, Guangzhou University, Guangzhou 510006, China \\ ${ }^{5}$ Key Laboratory of Mathematics and Interdisciplinary Sciences of Guangdong Higher Education Institutes, \\ Guangzhou University, Guangzhou 510006, China
}

Correspondence should be addressed to Wen-jun Yuan; wjyuan1957@126.com

Received 5 July 2014; Revised 18 September 2014; Accepted 2 October 2014; Published 14 October 2014

Academic Editor: Xinkai Chen

Copyright (c) 2014 Jian-ming Qi et al. This is an open access article distributed under the Creative Commons Attribution License, which permits unrestricted use, distribution, and reproduction in any medium, provided the original work is properly cited.

We employ the complex method to obtain the general meromorphic solutions of the Fisher equation, which improves the corresponding results obtained by Ablowitz and Zeppetella and other authors (Ablowitz and Zeppetella, 1979; Feng and Li, 2006; Guo and Chen, 1991), and $w_{g, i}(z)$ are new general meromorphic solutions of the Fisher equation for $c= \pm 5 i / \sqrt{6}$. Our results show that the complex method provides a powerful mathematical tool for solving great many nonlinear partial differential equations in mathematical physics.

\section{Dedicated to Professor Hongxun Yi 70th birthday}

\section{Introduction}

Consider the Fisher equation

$$
u_{t}=v u_{x x}+s u(1-u),
$$

which is a nonlinear diffusion equation as a model for the propagation of a mutant gene with an advantageous selection intensity $s$. It was suggested by Fisher as a deterministic version of a stochastic model for the spatial spread of a favored gene in a population in 1936.

Set $t^{\prime}=s t$ and $x^{\prime}=(s / v)^{1 / 2} x$ and drop the primes; (1) becomes

$$
u_{t}=u_{x x}+u(1-u) .
$$

Substituting the traveling wave transform $u(x, t)=w(z), x-$ $c t=z$ into (2) gives a nonlinear ordinary differential equation

$$
w^{\prime \prime}+c w^{\prime}+w(1-w)=0
$$

where $c$ is a constant.
Finding solutions of nonlinear models is a difficult and challenging task. Recently, the complex method was introduced by Yuan et al. [1-3].

In this paper, we employ the complex method to obtain the general solutions of (3). The general traveling wave exact solutions of the Fisher equation can be deduced by the traveling wave transform $u(x, t)=w(z), x-c t=z$. In order to state our results, we need some concepts and notations.

A meromorphic function $w(z)$ means that $w(z)$ is a holomorphic excepting for pole in the complex plane $\mathbb{C}$ except for poles. $\wp\left(z ; g_{2}, g_{3}\right)$ is the Weierstrass elliptic function with invariants $g_{2}$ and $g_{3}$. We say that a meromorphic function $f$ belongs to the class $W$ if $f$ is an elliptic function, or a rational function of $e^{\alpha z}, \alpha \in \mathbb{C}$, or a rational function of $z$.

Our main result is the following theorem.

Theorem 1. Equation (3) is integrable if and only if $c=0$, $\pm 5 / \sqrt{6}, \pm 5 i / \sqrt{6}$. Furthermore, the general solutions of (3) are of the forms below. 
(I) When $c=0$, the elliptic general solutions of (3) (see [1])

$$
w_{d}(z)=6\left\{-\wp(z)+\frac{1}{4}\left[\frac{\wp^{\prime}(z)+F}{\wp(z)-E}\right]^{2}\right\}-6 E+\frac{1}{2}
$$

where $g_{2}=1 / 12, F^{2}=4 E^{3}-g_{2} E-g_{3}, g_{3}$ and $E$ are arbitrary, in particular, which degenerates the simply periodic solutions

$$
w_{s}(z)=6 \alpha^{2} \operatorname{coth}^{2} \frac{\alpha}{2}\left(z-z_{0}\right)+\frac{1}{2} \alpha^{2}+\frac{1}{2},
$$

where $\alpha^{4}=1, z_{0} \in \mathbb{C}$.

(II) When $c= \pm 5 / \sqrt{6}$, the general solutions of (3) (see [4])

$$
w_{g}(z)=\exp \left\{\mp \frac{2 z}{\sqrt{6}}\right\} \wp\left(\exp \left\{\mp \frac{z}{\sqrt{6}}\right\}-s_{0} ; 0, g_{3}\right)
$$

where both $s_{0}$ and $g_{3}$ are arbitrary constants. In particular, the degenerate one-parameter family of solutions is given by

$$
w_{f}(z)=\frac{1}{\left\{1-\exp \left\{ \pm\left(z-z_{0}\right) / \sqrt{6}\right\}\right\}^{2}},
$$

where $z_{0} \in \mathbb{C}$.

(III) When $c= \pm 5 i / \sqrt{6}$, the general solutions of (3)

$$
w_{g, i}(z)=\exp \left\{\mp \frac{2 i z}{\sqrt{6}}\right\} \wp\left(i \exp \left\{\mp \frac{i z}{\sqrt{6}}\right\}-s_{0} ; 0, g_{3}\right)+1 \text {, }
$$

where both $s_{0}$ and $g_{3}$ are arbitrary constants. In particular, the degenerate one-parameter family of solutions is given by

$$
w_{f, i}(z)=-\frac{1}{\left\{1-\exp \left\{ \pm i\left(z-z_{0}\right) / \sqrt{6}\right\}\right\}^{2}}+1,
$$

where $z_{0} \in \mathbb{C}$.

Remark 2. The Fisher equation is classic and simplest case of the nonlinear reaction-diffusion equation, but there are many applications about it and many authors researched it [5]. The first explicit form of a traveling wave solution for the Fisher equation was obtained by Ablowitz and Zeppetella [4] using the Painlevé analysis. Many authors obtained only $w_{f, i}(z)$ by using other methods [5-7]; $w_{g, i}(z)$ are new general meromorphic solutions of the Fisher equation for $c=$ $\pm 5 i / \sqrt{6}$.

This paper is organized as follows. In the next section, the preliminary lemmas and the complex method are given. The proof of Theorem 1 is given and the general meromorphic solutions of (3) are derived by complex method in Section 3. Some conclusions and discussions are given in the final section.

\section{Preliminary Lemmas and the Complex Method}

In order to give complex method and the proof of Theorem 1, we need some notations and results.
Set $m \in \mathbb{N}:=\{1,2,3, \ldots\}, r_{j} \in \mathbb{N}_{0}=\mathbb{N} \cup\{0\}, r=$ $\left(r_{0}, r_{1}, \ldots, r_{m}\right)$, and $j=0,1, \ldots, m$. We define a differential monomial denoted by

$$
M_{r}[w](z):=[w(z)]^{r_{0}}\left[w^{\prime}(z)\right]^{r_{1}}\left[w^{\prime \prime}(z)\right]^{r_{2}} \cdots\left[w^{(m)}(z)\right]^{r_{m}} .
$$

$p(r):=r_{0}+r_{1}+\cdots+r_{m}$ is called the degree of $M_{r}[w]$. A differential polynomial $P\left(w, w^{\prime}, \ldots, w^{(m)}\right)$ is defined as follows

$$
P\left(w, w^{\prime}, \ldots, w^{(m)}\right):=\sum_{r \in I} a_{r} M_{r}[w]
$$

where $a_{r}$ are constants, and $I$ is a finite index set. The total degree of $P\left(w, w^{\prime}, \ldots, w^{(m)}\right)$ is defined by $\operatorname{deg} P\left(w, w^{\prime}, \ldots\right.$, $\left.w^{(m)}\right):=\max _{r \in I}\{p(r)\}$.

We will consider the following complex ordinary differential equations

$$
P\left(w, w^{\prime}, \ldots, w^{(m)}\right)=b w^{n}+c,
$$

where $b \neq 0, c$ are constants, $n \in \mathbb{N}$.

Let $p, q \in \mathbb{N}$. Suppose that (12) has a meromorphic solution $w$ with at least one pole, we say that (12) satisfies weak $\langle p, q\rangle$ condition if substituting Laurent series

$$
w(z)=\sum_{k=-q}^{\infty} c_{k} z^{k}, \quad q>0, c_{-q} \neq 0
$$

into (12) we can determine $p$ distinct Laurent singular parts below

$$
\sum_{k=-q}^{-1} c_{k} z^{k}
$$

In order to give the representations of elliptic solutions, we need some notations and results concerning elliptic function [8].

Let $\omega_{1}, \omega_{2}$ be two given complex numbers such that $\operatorname{Im}\left(\omega_{1} / \omega_{2}\right)>0, L=L\left[2 \omega_{1}, 2 \omega_{2}\right]$ be discrete subset $L\left[2 \omega_{1}, 2 \omega_{2}\right]=\left\{\omega \mid \omega=2 n \omega_{1}+2 m \omega_{2}, n, m \in \mathbb{Z}\right\}$, which is isomorphic to $\mathbb{Z} \times \mathbb{Z}$. The discriminant $\Delta=\Delta\left(c_{1}, c_{2}\right):=$ $c_{1}^{3}-27 c_{2}^{2}$ and

$$
s_{n}=s_{n}(L):=\sum_{\omega \in L \backslash\{0\}} \frac{1}{\omega^{n}} .
$$

Weierstrass elliptic function $\wp(z):=\wp\left(z, g_{2}, g_{3}\right)$ is a meromorphic function with double periods $2 \omega_{1}, 2 \omega_{2}$ and satisfying the equation

$$
\left(\wp^{\prime}(z)\right)^{2}=4 \wp(z)^{3}-g_{2} \wp(z)-g_{3}
$$

where $g_{2}=60 s_{4}, g_{3}=140 s_{6}$, and $\Delta\left(g_{2}, g_{3}\right) \neq 0$.

By changing (16) to the form

$$
\left(\wp^{\prime}(z)\right)^{2}=4\left(\wp(z)-e_{1}\right)\left(\wp(z)-e_{2}\right)\left(\wp(z)-e_{2}\right),
$$

we have $e_{1}=\wp\left(\omega_{1}\right), e_{2}=\wp\left(\omega_{2}\right), e_{3}=\wp\left(\omega_{1}+\omega_{2}\right)$.

Inversely, given two complex numbers $g_{2}$ and $g_{3}$ such that $\Delta\left(g_{2}, g_{3}\right) \neq 0$, then there exists double periods $2 \omega_{1}, 2 \omega_{2}$ Weierstrass elliptic function $\wp(z)$ such that above results hold. 
Lemma 3 (see $[8,9]$ ). Weierstrass elliptic functions $\wp(z):=$ $\wp\left(z, g_{2}, g_{3}\right)$ have two successive degeneracies and addition formula:

(I) degeneracy to simply periodic functions (i.e., rational functions of one exponential $e^{k z}$ ) according to

$$
\wp\left(z, 3 d^{2},-d^{3}\right)=2 d-\frac{3 d}{2} \operatorname{coth}^{2} \sqrt{\frac{3 d}{2}} z,
$$

if one root $e_{j}$ is double $\left(\Delta\left(g_{2}, g_{3}\right)=0\right)$,

(II) degeneracy to rational functions of $z$ according to

$$
\wp(z, 0,0)=\frac{1}{z^{2}}
$$

if one root $e_{j}$ is triple $\left(g_{2}=g_{3}=0\right)$,

(III) addition formula

$$
\wp\left(z-z_{0}\right)=-\wp(z)-\wp\left(z_{0}\right)+\frac{1}{4}\left[\frac{\wp^{\prime}(z)+\wp^{\prime}\left(z_{0}\right)}{\wp(z)-\wp\left(z_{0}\right)}\right]^{2} .
$$

By above lemma and results, we can give a new method below, say complex method, to find exact solutions of some PDEs.

Step 1. Substituting the transform $T: u(x, t) \rightarrow w(z)$, $(x, t) \rightarrow z$ into a given PDE gives a nonlinear ordinary differential equations (12).

Step 2. Substitute (13) into (12) to determine that weak $\langle p, q\rangle$ condition holds, and pass the Painlevé test for (12).

Step 3. Find the meromorphic solutions $w(z)$ of (12) with pole at $z=0$, which have $m-1$ integral constants.

Step 4. By Lemma 3, we obtain the general meromorphic solutions $w\left(z-z_{0}\right)$.

Step 5. Substituting the inverse transform $T^{-1}$ into these meromorphic solutions $w\left(z-z_{0}\right)$, then we get all exact solutions $u(x, t)$ of the original given PDE.

\section{Proof of Theorem 1}

Substituting (13) into (3), we have $q=2, p=1, c_{-2}=6$, $c_{-1}=-6 c / 5, c_{0}=-c^{2} / 50+1 / 2, c_{1}=-c^{3} / 250, c_{2}=1 / 40-$ $189 c^{4} / 135000, c_{3}=891 c / 48600-6399 c^{5} / 6075000$, and

$$
0 \times c_{4}+\frac{5}{2}\left(\frac{c}{5}\right)^{2}-90\left(\frac{c}{5}\right)^{6}=0
$$

For the Laurent expansion (13) to be valid $c$ satisfies this equation and $c_{4}$ is an arbitrary constant. Therefore, $c=0$ or $c= \pm 5 / \sqrt{6}$ or $c= \pm 5 i / \sqrt{6}$, where $i^{2}=-1$. For other $c$ it would be necessary to add logarithmic terms to the expansion, thus giving a branch point rather than a pole. For $c=0$, the solution of (3) has been given in Theorem 1 and can be also found by direct integration.
For $c= \pm 5 / \sqrt{6}$, (3) is integrable by Ablowitz and Zeppetella [4] using the Painlevé analysis and the general solutions were given. That is, when $c= \pm 5 / \sqrt{6}$, the general solutions of (3)

$$
w_{g}(z)=\exp \left\{\mp \frac{2 z}{\sqrt{6}}\right\} \wp\left(\exp \left\{\mp \frac{z}{\sqrt{6}}\right\}-s_{0} ; 0, g_{3}\right)
$$

where both $s_{0}$ and $g_{3}$ are arbitrary constants, in particular, which degenerates the one-parameter family of solutions

$$
w_{f}(z)=\frac{1}{\left\{1-\exp \left\{ \pm\left(z-z_{0}\right) / \sqrt{6}\right\}\right\}^{2}}
$$

where $z_{0} \in \mathbb{C}$.

Thus we consider only for cases $c= \pm 5 i / \sqrt{6}$, where $i^{2}=$ -1 . By the same arguments of Ablowitz and Zeppetella, we transform (3) with $c= \pm 5 i / \sqrt{6}$ into the first Painlevé type equation. In this way we find the general solutions.

Setting $w(z)=f(z) u(s)+1, s=g(z)$, and substituting in Fisher's equation (3), we obtain that the equation for $u(s)$ is

$$
u^{\prime \prime}=6 u^{2}
$$

where

$$
f(z)=\exp \left\{\mp \frac{2 i z}{\sqrt{6}}\right\}, \quad g(z)=i \exp \left\{\mp \frac{i z}{\sqrt{6}}\right\} .
$$

The general solutions of (24) are the Weierstrass elliptic functions $u(s)=\wp\left(s-s_{0} ; 0, g_{3}\right)$, where $s_{0}$ and $g_{3}$ are two arbitrary constants.

Therefore, when $c= \pm 5 i / \sqrt{6}$, the general solutions of (3)

$$
w_{g, i}(z)=\exp \left\{\mp \frac{2 i z}{\sqrt{6}}\right\} \wp\left(i \exp \left\{\mp \frac{i z}{\sqrt{6}}\right\}-s_{0} ; 0, g_{3}\right)+1 \text {, }
$$

where both $s_{0}$ and $g_{3}$ are arbitrary constants. In particular, by Lemma 3 and $g_{3}=0, w_{g, i}(z)$ degenerate the one-parameter family of solutions

$$
w_{f, i}(z)=-\frac{1}{\left\{1-\exp \left\{ \pm i\left(z-z_{0}\right) / \sqrt{6}\right\}\right\}^{2}}+1,
$$

where $z_{0} \in \mathbb{C}$.

\section{Conclusions}

Complex method is a very important tool in finding the exact solutions of nonlinear evolution equations, and the Fisher equation is classic and simplest case of the nonlinear reactiondiffusion equation. In this paper, we employ the complex method to obtain the general meromorphic solutions of the Fisher equation, which improves the corresponding result obtained by Ablowitz and Zeppetella and other authors [4$6]$, and $w_{g, i}(z)$ are new general meromorphic solutions of the Fisher equation for $c= \pm 5 i / \sqrt{6}$. Our results show that the complex method provides a powerful mathematical tool for solving great many nonlinear partial differential equations in mathematical physics. 


\section{Conflict of Interests}

The authors declare that there is no conflict of interests regarding the publication of this paper.

\section{Acknowledgments}

The authors appreciate reviewers very much for their positive and constructive comments and suggestions on their paper. This work was supported by Tianyuan Youth Fund of the NSF of China (11326083), the NSF of China (11171184, 11271090), Shanghai University Young Teacher Training Program (ZZSDJ12020), Innovation Program of Shanghai Municipal Education Commission (14YZ164), NSF of Guangdong Province (S2012010010121), and projects 13XKJC01 from the Leading Academic Discipline Project of Shanghai Dianji University.

\section{References}

[1] W. Yuan, Y. Li, and J. Lin, "Meromorphic solutions of an auxiliary ordinary differential equation using complex method," Mathematical Methods in the Applied Sciences, vol. 36, no. 13, pp. 1776-1782, 2013.

[2] W. Yuan, Y. Huang, and Y. Shang, "All traveling wave exact solutions of two nonlinear physical models," Applied Mathematics and Computation, vol. 219, no. 11, pp. 6212-6223, 2013.

[3] W. J. Yuan, Y. D. Shang, Y. Huang, and H. Wang, “The representation of meromorphic solutions of certain ordinary differential equations and its applications," Scientia Sinica Mathematica, vol. 43, pp. 563-575, 2013 (Chinese).

[4] M. J. Ablowitz and A. Zeppetella, "Explicit solutions of Fisher's equation for a special wave speed," Bulletin of Mathematical Biology, vol. 41, no. 6, pp. 835-840, 1979.

[5] Z. Feng and Y. Li, "Complex traveling wave solutions to the Fisher equation," Physica A: Statistical Mechanics and its Applications, vol. 366, pp. 115-123, 2006.

[6] B. Y. Guo and Z. X. Chen, "Analytic solutions of the Fisher equation," Journal of Physics A: Mathematical and General, vol. 24, no. 3, pp. 645-650, 1991.

[7] H. C. Rosu and O. Cornejo-Pérez, "Supersymmetric pairing of kinks for polynomial nonlinearities," Physical Review E: Statistical, Nonlinear, and Soft Matter Physics, vol. 71, no. 4, Article ID 046607, 7 pages, 2005.

[8] S. Lang, Elliptic Functions, Springer, New York, NY, USA, 2nd edition, 1987.

[9] R. Conte and M. Musette, "Elliptic general analytic solutions," Studies in Applied Mathematics, vol. 123, no. 1, pp. 63-81, 2009. 


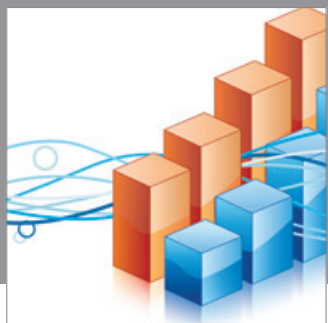

Advances in

Operations Research

mansans

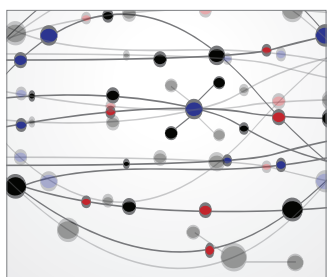

The Scientific World Journal
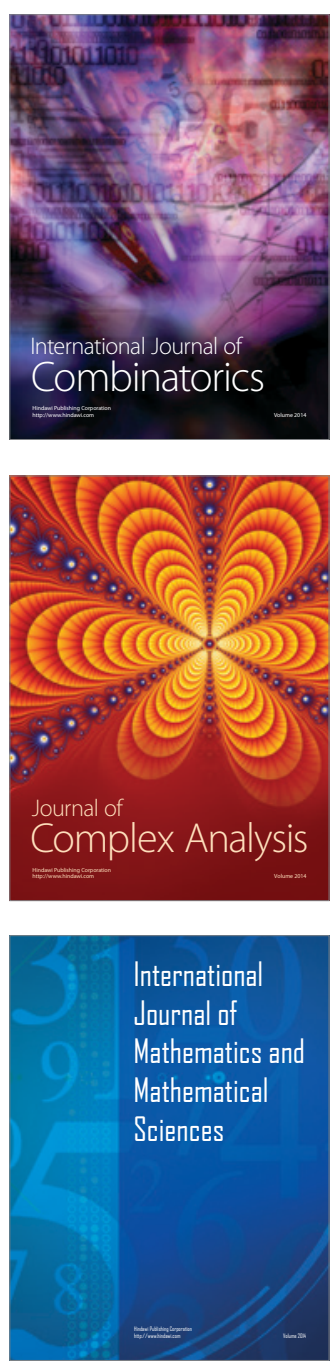
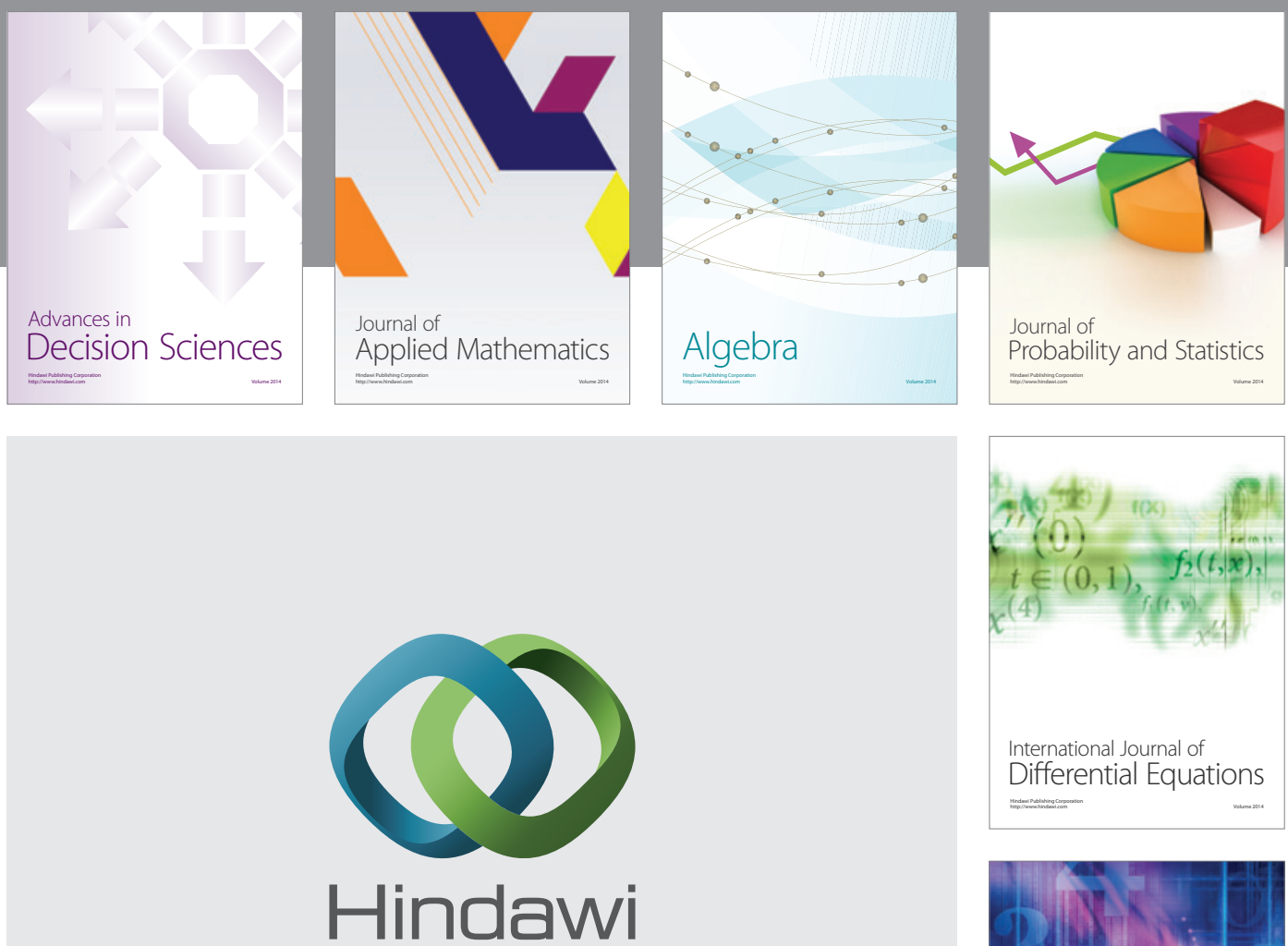

Submit your manuscripts at http://www.hindawi.com
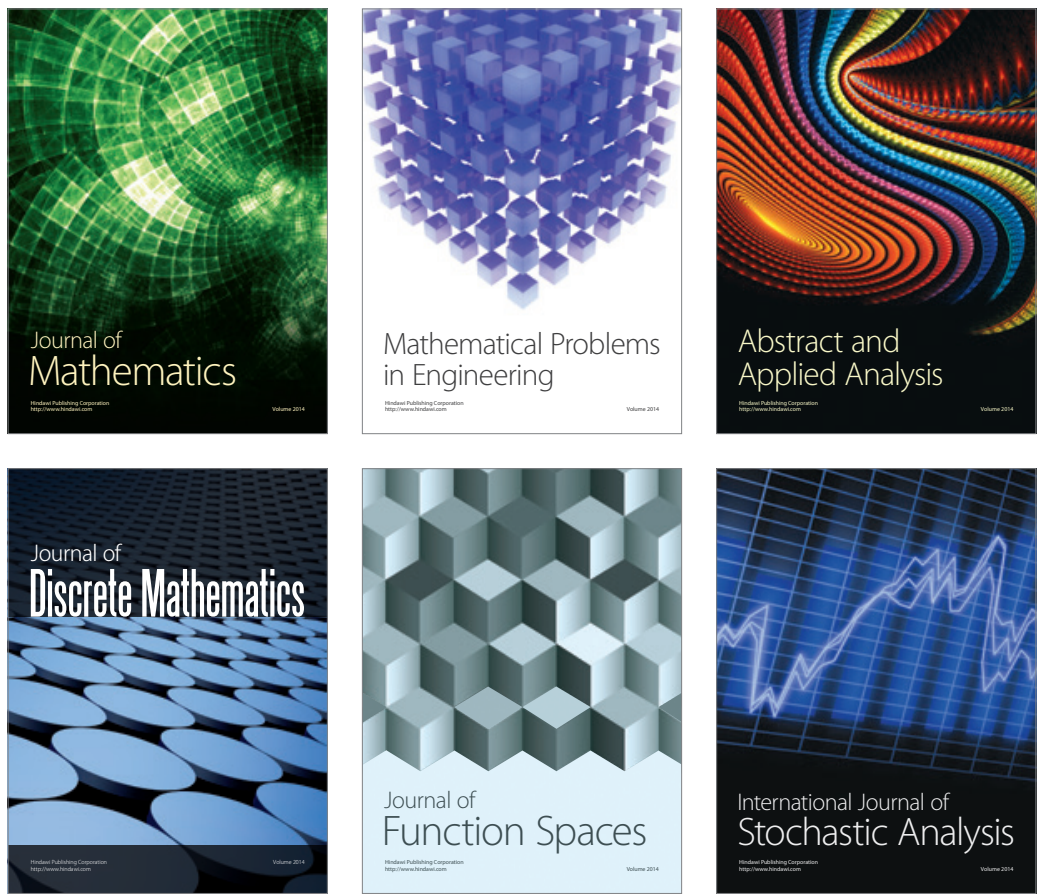

Journal of

Function Spaces

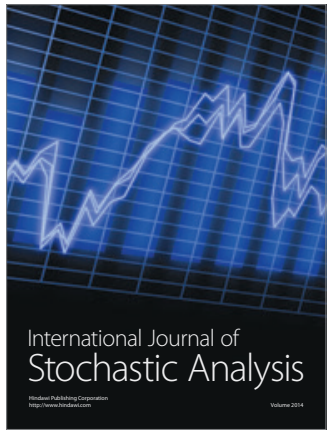

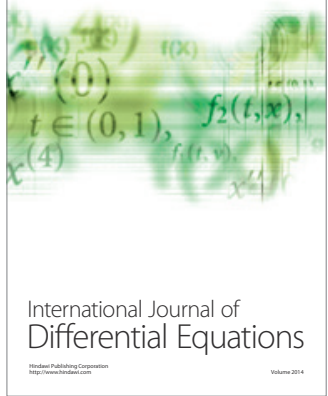
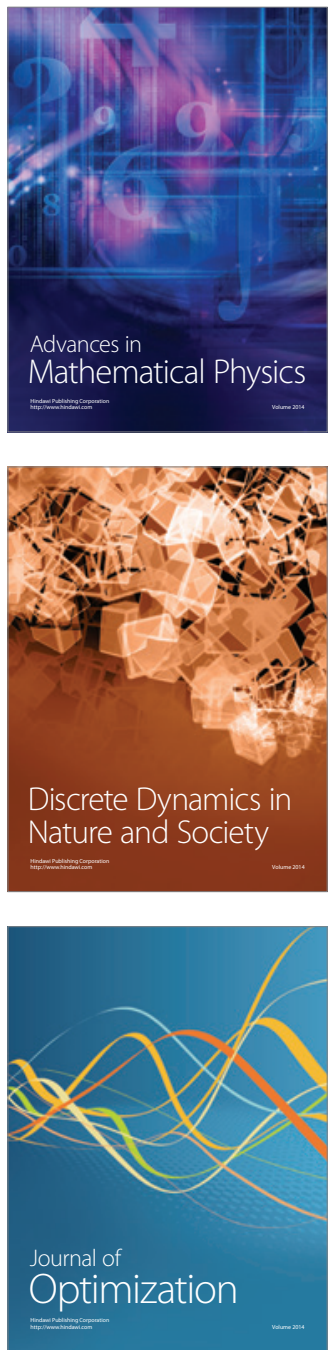